\title{
Derivation rings of Lie rings
}

\section{Orest D. Artemovych ${ }^{1}$}

\begin{abstract}
We establish some connections between Lie rings, their derivation rings
\end{abstract} and generalized derivations rings.

Keywords Lie ring $\cdot$ Derivation $\cdot$ Generalized derivation $\cdot$ Lie $F C$-ring

Mathematics Subject Classification 17B40 - 17B20

\section{Introduction}

Let $A$ be a Lie ring (with addition "+" and multiplication " $[-,-]$ "). An additive mapping $d: A \rightarrow A$ is called a derivation of $A$ if

$$
d([x, y])=[d(x), y]+[x, d(y)]
$$

for any $x, y \in A$. By Der $A$ we denote the set of all derivations of $A$. It is well known that $\operatorname{Der} A$ is a Lie ring with respect to operations of the point-wise addition " + " and the point-wise Lie multiplication " $[-,-]$ ” defined by rules

$$
(d+\delta)(r)=d(r)+\delta(r) \text { and }[d, \delta](r)=d(\delta(r))-\delta(d(r))
$$

$\varangle$ Orest D. Artemovych artemo@usk.pk.edu.pl

1 Institute of Mathematics, Cracow University of Technology, ul. Warszawska 24, 31-155 Cracow, Poland 
for all $r \in A$ and $d, \delta \in \operatorname{Der} A$ [11]. The mapping

$$
\operatorname{ad}_{a}: A \ni x \mapsto[a, x] \in A
$$

determines a derivation $\operatorname{ad}_{a}$ of $A$ (so-called inner derivation of $A$ induced by $a \in A$ ). The set

$$
\operatorname{ad} A=\left\{\operatorname{ad}_{a} \mid a \in A\right\}
$$

of all inner derivations of $A$ is an ideal in $\operatorname{Der} A$.

Let $\mathbf{N}$ be the set of positive integers,

$$
\left[a_{1}, \ldots, a_{n}, a_{n+1}\right]:=\left[\left[a_{1}, \ldots, a_{n}\right], a_{n+1}\right]
$$

for any $n \in \mathbf{N}$ and $a_{1}, \ldots, a_{n}, a_{n+1} \in A$. If $A_{i} \subseteq A$, then $\left[A_{1}, \ldots, A_{n}\right]$ is a subgroup of the additive group $A^{+}$of $A$ generated by all $\left[a_{1}, \ldots, a_{n}\right]$, where $a_{i} \in A_{i} \quad(i=$ $1, \ldots, n)$. A Lie ring $A$ is called:

- solvable if there exists $n \in \mathbf{N}$ such that $A^{(n)}=0$, where $A^{(1)}:=[A, A]=A^{\prime}$, $A^{(2)}:=\left[A^{\prime}, A^{\prime}\right]=A^{\prime \prime}$ and $A^{(k+1)}=\left[A^{(k)}, A^{(k)}\right]$ for any $k \in \mathbf{N}$,

- abelian if $A^{\prime}=0$,

- perfect if $A^{\prime}=A$,

- complete if its derivations are inner and the center $Z(A)=0$ is zero (i.e. $A$ is centerless) [13].

Many authors have been investigated the structure of derivation algebra $\operatorname{Der} L$ and its relations with the structure of a (finite or infinite dimensional) Lie algebra $L$ (see e.g. $[6,7,9,12,23-26,28,30,31]$ and others). In this way Leger [16] has investigated Lie algebras $L$ over a field of characteristic 0 such that $\operatorname{Der} L=\operatorname{ad} L$. Luks [20] has constructed an example of a Lie algebra over a field of characteristic zero with only inner derivations which is not complete. Su and Zhu [27, Theorem 1.1] have proved that the Lie algebra of all derivations of a centerless perfect Lie algebra (over any field) is complete. Augolopoulos [1] has constructed a class of complete Lie algebras over the complex numbers field that are not semisimple. Interesting results about complete Lie algebras were obtained by Meng and Wang (see [21], where further references can be found).

An additive mapping $F: A \rightarrow A$ is called a generalized derivation of $A$ associated with a derivation $\delta \in \operatorname{Der} A$ (in the sence of Brešar [4]) if

$$
F([x, y])=[F(x), y]+[x, \delta(y)]
$$

for any $x, y \in A$. The set of all generalized derivations of a Lie ring $A$ we denote by

GDer $A$.

We will write $(F, \delta) \in \operatorname{GDer} A$ if and only if $F$ is a generalized derivation of $A$ associated with $\delta \in \operatorname{Der} A$. Since $(\delta, \delta) \in \operatorname{GDer} A$ for any $\delta \in \operatorname{Der} A$, we conclude 
that

$$
\operatorname{ad} A \subseteq \operatorname{Der} A \subseteq \operatorname{GDer} A
$$

A generalized derivation $F$ of $A$ associated with an inner derivation $\operatorname{ad}_{a} \in \operatorname{ad} A$ is called a generalized inner derivation of $A$. By

\section{IGDer $A$}

we denote the set of all generalized inner derivations of $A$. Another types of generalized derivations was introduced in [8-10,17,29,32] and others.

We shall use the following notation. Let $D=\operatorname{Der} A, G=\operatorname{GDer} A, \Delta$ be a nonempty subset of $D$ (respectively $G$ ). If $I \subseteq A$ and $\delta(I) \subseteq I$ for all $\delta \in \Delta$, then we say that $I$ is $\Delta$-closed in $A$. If $I$ is a $\Delta$-closed ideal of $A$, then it is called a $\Delta$-ideal of $A$. Moreover, $Z(A):=\{z \in A \mid z a=a z$ for all $a \in A\}$ is the center of $A$.

Let $X \in\{0, \Delta\}$. By a 0 -ideal of $R$ we mean an ideal of $R$. An ideal $Y$ of a Lie ring $A$ is called:

- $X$-semiprime if, for any $X$-ideal $B$ of $A$, the condition $[B, B] \subseteq Y$ implies that $B \subseteq Y$

- $X$-prime if, for any $X$-ideals $B, C$ of $A$, the condition $[B, C] \subseteq Y$ implies that $B \subseteq Y$ or $C \subseteq Y$,

- $X$-simple if $[\bar{A}, A] \nsubseteq Y$ and, for any proper $X$-ideal $B$ of $A$, it is true that $B \subseteq Y$,

- $X$-primary if, for any $X$-ideals $B, C$ of $A$, the condition $[B, C] \subseteq Y$ implies that $B \subseteq Y$ or

$$
[\underbrace{C, \ldots, C}_{m \text { times }}] \subseteq Y
$$

for some $m \in \mathbf{N}$.

In particular, if the zero ideal 0 of $A$ is a $X$-simple (respectively $X$-prime, $X$-semiprime or $X$-primary) Lie ring and $X=0$, then $A$ is called simple (respectively prime, semiprime or primary). Moreover, $A / Y$ is semiprime (respectively prime, simple or primary) if so is $Y$. Every $\Delta$-prime Lie ring is $\Delta$-semiprime and every $\Delta$-simple Lie ring is $\Delta$-prime.

The purpose of this paper is to study relationships between Lie rings $A$, their derivation rings $\operatorname{Der} A$ (in particular, inner derivation $\operatorname{rings} \operatorname{IDer} A$ ) and generalized derivation rings GDer $A$. In many cases every derivation of a simple Lie algebra is inner (see e.g. [11,24,34,35] and others). Our first result is the following

Proposition 1 Let A be a Lie ring. Then we have:

(1) if D is a simple Lie ring, then one of the following holds:

(a) $A$ is abelian,

(b) $D=\operatorname{ad} A, A / Z(A)$ is a simple Lie ring and either

(i) $A=A^{\prime}$ is simple, or

(ii) $A=A^{\prime}+Z(A)$ and $A^{\prime}$ is the smallest noncentral ideal of $A$,

(2) if $A$ is a $D$-simple Lie ring, then $A=A^{\prime}, Z(A)=0$ and $D$ is complete. 
We say that a subring $S$ is of finite index in $A$ if its additive subgroup $S^{+}$has finite index $|A: S|:=\left|A^{+}: S^{+}\right|$in $A^{+}$. By analogy with group theory [22], we will say that a Lie ring $A$ is $a$ Lie FC-ring if, for any $a \in A$, the centralizer

$$
C_{A}(a)=\{z \in A \mid[z, a]=0\}
$$

is of finite index in $A$. If $A$ is $F C$, then for every $a \in A$ there exists an ideal $I_{a}$ of $A$ such that $\left[a, I_{a}\right]=0$ (Corollary 4 ). In the proof of this result we use the following

Proposition 2 Let $A$ be a Lie ring. If $S$ is its subring of finite index, then there exists an ideal $I$ of $A$ such that $I \leq S$ and $|A: I|<\infty$.

Since an inner derivation $\operatorname{ad}_{a}: A^{+} \rightarrow A^{+}$is an endomorphism of the additive group $A^{+}$for any $a \in A$,

$$
[A, a] \ni[x, a] \mapsto x+C_{A}(a) \in A / C_{A}(a)
$$

is an additive group isomorphism and the kernel $\operatorname{Ker} \operatorname{ad}_{a}=C_{A}(a)$, we conclude that $A$ is $F C$ if and only if the image $\operatorname{Im~ad}_{a}$ is finite for any $a \in A$. If the set $\operatorname{IDer} A$ is finite (or equivalently $|A: Z(A)|<\infty$ by Proposition 3), then $A$ is $F C$ (Lemma 6), the commutator ideal $A^{\prime}$ is finite and there exists a solvable ideal $S$ of $A$ such that $S \leq A^{\prime}, A^{\prime}=A^{\prime \prime}+S$ and $A^{\prime} / S$ is a direct summand of $A / S$ (Corollary 6).

Derivations are very important in the study of structures of Lie algebras. Lie algebras with semisimple (in particular, simple) derivation algebras have been discussed by Hochschild [9], Block [3], de Ruiter [5], Elduque and Montaner [7], Walcher [34] and others. For example, it was proved in [9, Theorem 4.4] that a finite dimensional Lie algebra $L$ over a field of characteristic 0 is semisimple if and only if its derivation algebra $\operatorname{Der} L$ is semisimple. In this way we prove the next result.

Theorem 1 Let $A$ be a Lie ring. If $A$ is a D-prime (respectively D-semiprime) Lie ring, then $D$ is prime (respectively semiprime).

An additive mapping $T: A \rightarrow A$ is called $a$ multiplier of $A$ if

$$
T([x, y])=[T(x), y]
$$

for all $x, y \in A$. Then we have

$$
T([x, y])=T(-[y, x])=-[T(y), x]=[x, T(y)] .
$$

The set of all multipliers of $A$ we denote by

$$
\mathrm{M}(A) \text {. }
$$

Obviously, for any $T \in \mathrm{M}(A),(T, 0) \in \operatorname{GDer} A$ and so

$$
\mathrm{M}(A) \subseteq \operatorname{IGDer} A
$$

We obtain the following 
Theorem 2 Let $A$ be a Lie ring. If $\mathrm{GDer} A / \mathrm{M}(A)$ is a prime (respectively semiprime, simple or primary) Lie ring, then $Z(A)$ is a $G$-prime (respectively $G$-semiprime, $G$-simple or $G$-primary) ideal of A.

Any unexplained terminology is standard as in [13-15,22,33].

\section{Inner derivations}

We first give some information about inner derivation rings.

Lemma 1 Let $A$ be a Lie ring. Then we have:

(i) if $\Delta$ is a subring of $\operatorname{Der} A, S$ is a $\Delta$-closed additive subgroup of $A$ and $\operatorname{ad}_{S} A=$ $\left\{\operatorname{ad}_{t} \mid t \in S\right\}$, then $\left[\operatorname{ad}_{S} A, \Delta\right] \subseteq \operatorname{ad}_{S} A\left(\right.$ where $\left.\operatorname{ad}_{A} A=\operatorname{ad} A\right)$,

(ii) if $K$ is an ideal of $A$, then $\operatorname{ad}_{K} A$ is an ideal of $\operatorname{ad} A$,

(iii) if $\Phi$ is an ideal of $\operatorname{Der} A$, then

$$
\nabla_{\Phi}=\left\{x \in A \mid \operatorname{ad}_{x} \in \Phi\right\}
$$

is a D-ideal of $A$,

(iv) if $\Phi$ is an ideal of $\operatorname{ad} A$, then $\nabla_{\Phi}$ is an ideal of $A$,

Proof (i) If $s \in S$ and $\delta \in \Delta$, then $\delta(s) \in S$ and so

$$
\left[\delta, \operatorname{ad}_{s}\right]=\operatorname{ad}_{\delta(s)} \in \operatorname{ad}_{S} A .
$$

(ii) Since $K$ is an $(\operatorname{ad} A)$-closed additive subgroup of $A$, the result follows from part (i).

(iii) If $x, y \in \nabla_{\Phi}, a \in A$ and $\delta \in D$, then $\operatorname{ad}_{x-y}=\operatorname{ad}_{x}-\operatorname{ad}_{y}, \operatorname{ad}_{[a, x]}=$ $\left[\operatorname{ad}_{a}, \operatorname{ad}_{x}\right], \operatorname{ad}_{\delta(x)}=\left[\delta, \operatorname{ad}_{x}\right] \in \Phi$ and consequently $x-y,[a, x], \delta(x) \in \nabla_{\Phi}$.

(iv) By the same argument as in part (iii).

Lemma 2 Let $A$ be a Lie ring and $\Delta$ a subring of Der $A$. Then we have:

(i) if $B$ is a $\Delta$-ideal of $A$, then $\{\delta \in \Delta \mid \delta(A) \subseteq B\}$ is an ideal of $\Delta$,

(ii) if $B$ is an ideal of $A$, then $\{\delta \in \operatorname{ad} A \mid \delta(A) \subseteq B\}$ is an ideal of $\operatorname{ad} A$,

(iii) if $\Phi$ is an ideal of $\operatorname{ad} A$, then $\{a \in A \mid \delta(a)=0$ for all $\delta \in \Phi\}$ is an ideal of $A$,

(iv) if $B$ is a $\Delta$-ideal of $A$, then $\left\{x \in A \mid \operatorname{ad}_{x}(A) \subseteq B\right\}$ is a $\Delta$-ideal of $A$,

(v) if $B$ is a $\Delta$-ideal of $A$, then the centralizer $C_{A}(B)=\left\{x \in A \mid \operatorname{ad}_{x}(B)=0\right\}$ of $B$ in $A$ is a $\Delta$-ideal of $A$,

(vi) [19, Proposition 2.2] there exists the Lie ring isomorphism

$$
\operatorname{ad} A \ni \operatorname{ad}_{a} \mapsto a+Z(A) \in A / Z(A)
$$

(vii) if $B$ is a $\Delta$-ideal of $A$, then the center $Z(B)$ is a $\Delta$-ideal of $A$.

Proof By routine calculations. 
Lemma 3 Let $A$ be a $\Delta$-semiprime Lie ring, $B$ its nonzero $\Delta$-ideal, where $\emptyset \neq \Delta \subseteq$ $D$. Then the following are true:

(i) A is nonabelian,

(ii) $Z(A)=0$, i.e. $A$ is centerless,

(iii) $B \cap C_{A}(B)=0$,

(iv) $Z(B)=0$,

(v) $C_{A}\left(A^{\prime}\right)=0$,

(vi) if $A$ is $\Delta$-prime, then $C_{A}(B)=0$.

Proof (i)-(ii) Evident.

(iii) Inasmuch as $B \cap C_{A}(B)$ is a $\Delta$-ideal of $A$ in view of Lemma 3 (v) and

$$
\left[B \bigcap C_{A}(B), B \bigcap C_{A}(B)\right]=0
$$

we deduce that $B \cap C_{A}(B)=0$.

(iv) In view of Lemma 2 (vii), $[A, Z(B)]$ is a $\Delta$-ideal of $A$ and $[A, Z(B)] \subseteq Z(B)$. However,

$$
[[A, Z(B)],[A, Z(B)]]=0
$$

and therefore $[A, Z(B)]=0$. Then we find that $Z(B) \subseteq Z(A)=0$.

(v) It is easy to see that $A^{\prime}$ is nonzero,

$$
[z, A] \subseteq A^{\prime} \bigcap C_{A}\left(A^{\prime}\right)=0
$$

for any $z \in C_{A}\left(A^{\prime}\right)$ and so $z \in Z(A)$. Hence $z=0$ by (ii).

(vi) It holds in view of Lemma 2 (v).

Corollary 1 Let A be a Lie ring. Then we have:

(i) if $A$ is simple, then $\operatorname{ad} A$ is a simple Lie ring,

(ii) if $\operatorname{ad} A$ is simple, then $A / Z(A)$ is a simple Lie ring and

$$
A=A^{\prime}+Z(A)
$$

(and then $A^{\prime}$ is the smallest noncentral ideal of $A$ ),

(iii) if $A$ is a prime (respectively semiprime) Lie ring, then so is $\operatorname{ad} A$,

(iv) if $A / Z(A)$ is a primary Lie ring, then so is $\operatorname{ad} A$,

(v) if $\operatorname{ad} A$ is prime (respectively semiprime or primary), then so is $A / Z(A)$.

Proof (i) and (iii)-(v) It follows in view of Lemma 2 (vi).

(ii) Obviously that $A$ is nonabelian and therefore $A^{\prime} \neq 0$. Lemma 2 (vi) implies that the quotient Lie ring $A / Z(A)$ is simple. Using the fact that

$$
\operatorname{ad}_{A^{\prime}} A=[\operatorname{ad} A, \operatorname{ad} A]=\operatorname{ad} A
$$

and $A^{\prime} \nsubseteq Z(A)$ we deduce that $A$ satisfies Eq. (2). 
Corollary 2 Let A be a semiprime Lie ring. Then adA is simple if and only if so is A.

Let $p$ be a prime,

$$
F(A):=\left\{a \in A \mid a \text { is of finite order in the additive group } A^{+}\right\}
$$

the torsion part and

$$
F_{p}(A):=\left\{a \in F(A) \mid p^{n} a=0 \text { for some nonnegative integer } n\right\}
$$

the torsion $p$-part of a Lie ring $A$.

Remark 1 If $A$ is a $\Delta$-prime Lie ring, then one of the following holds:

(i) $F(A)=0$,

(ii) $p A=0$ for some prime $p$.

Indeed, if $F(A)$ is nonzero, then $F_{p}(A) \neq 0$ for some prime $p$. From $p A \neq 0$ it follows that

$$
\Omega_{1}:=\left\{a \in F_{p}(A) \mid p a=0\right\} \neq 0
$$

and $\left[p A, \Omega_{1}\right]=0$, a contradiction. Hence $p A=0$.

Lemma 4 Let $A$ be a centerless Lie ring. If $\Phi$ is an ideal of $\operatorname{Der} A$, then

$$
\Phi \bigcap \operatorname{ad} A=0 \Leftrightarrow \Phi=0
$$

Proof In fact, if $\Phi \cap \operatorname{ad} A=0$, then $0=\left[d, \operatorname{ad}_{a}\right]=\operatorname{ad}_{d(a)}$ and therefore $d(a) \in Z(A)$ for any $d \in \Phi$ and $a \in A$. Consequently $d=0$.

Lemma 5 If $A$ is a $D$-simple Lie ring, then

$$
\operatorname{ad} A=[\operatorname{ad} A, \operatorname{ad} A]
$$

is the smallest nonzero ideal of $D$ (and so A is perfect).

Proof It is easy to see that $A^{\prime}$ is a nonzero $D$-ideal of $A, Z(A)=0$ and therefore $A^{\prime}=A$ by Corollary 1 . Let $\Phi$ be a nonzero ideal of $D$. By Lemma 4,

$$
\Phi_{1}:=\Phi \bigcap \operatorname{ad} A \neq 0
$$

Then $\nabla_{\Phi_{1}} \neq 0$ is a $D$-ideal of $A$ by Lemma $1($ ii i $)$ and consequently $\nabla_{\Phi_{1}}=A$. Then $\operatorname{ad} A \subseteq \Phi$, Eqs. (4) and (3) are true and $\operatorname{ad} A$ is the smallest nonzero ideal of $D$.

Corollary 3 Let A be a Lie ring. Then the following hold: 
(1) $[\operatorname{ad} A, \operatorname{ad} A]$ is a simple (respectively semiprime, prime or primary) Lie ring if and only if so is

$$
A^{\prime} /\left(A^{\prime} \bigcap Z(A)\right)
$$

(2) if $A$ is semiprime and $[\operatorname{ad} A, \operatorname{ad} A]$ is a simple Lie ring, then $A^{\prime}$ is the smallest nonzero ideal of $A$.

Proof (1) If $a, b \in A$, then the rule

$$
[\operatorname{ad} A, \operatorname{ad} A] \ni\left[\operatorname{ad}_{a}, \operatorname{ad}_{b}\right] \mapsto[a, b]+\left(A^{\prime} \bigcap Z(A)\right) \in A^{\prime} /\left(A^{\prime} \bigcap Z(A)\right)
$$

induces a Lie ring isomorphism.

(2) Let $I$ be a nonzero ideal of $A$. Then $0 \neq[I, I] \subseteq A^{\prime}$. Since $\operatorname{ad}_{I} A$ is a nonzero ideal of the Lie $\operatorname{ring} \operatorname{ad} A$, we deduce that

$$
\operatorname{ad}_{[I, I]} A=\left[\operatorname{ad}_{I} A, \operatorname{ad}_{I} A\right]=[\operatorname{ad} A, \operatorname{ad} A]=\operatorname{ad}_{A^{\prime}} A .
$$

Moreover, $Z(A)=0$ and therefore $A^{\prime}=[I, I] \subseteq I$. Hence $A^{\prime}$ is the smallest nonzero ideal of $A$.

\section{Lie $F C$-rings}

Proposition 2 is analogous with the Lewin result [18, Lemma 1].

Proof of Proposition 2. Since every Lie ring is a Leibniz ring, Proposition 2 follows from [2, Proposition 5.2]. We prove it here in order to have the paper more selfcontained. Suppose that $|A: S|=n$ for some $n \in \mathbf{N}$ and the quotient group

$$
A^{+} / S^{+}=\left\{a_{1}+S^{+}, \ldots, a_{n}+S^{+}\right\}
$$

for some elements $a_{1}, \ldots, a_{n} \in A$. Let $s \in S$. The rule

$$
g_{s}: A^{+} / S^{+} \ni a+S^{+} \mapsto[a, s]+S^{+} \in A^{+} / S^{+}
$$

determines an endomorphism $g_{S}$ of the additive group $A^{+} / S^{+}$. Since $A^{+} / S^{+}$is finite, its endomorphism ring $\operatorname{End}\left(A^{+} / S^{+}\right)$is the ones. Then the group homomorphism

$$
g: S^{+} \ni s \mapsto g_{s} \in \operatorname{End}\left(A^{+} / S^{+}\right)
$$

has the kernel $K_{g}:=\{s \in S \mid[A, s] \subseteq S\}$ of finite index in $S$. The rule

$$
\varphi_{\left(i_{1}, \ldots, i_{k}\right)}: K_{g} \ni w \mapsto \varphi_{\left(w, i_{1}, \ldots, i_{k}\right)} \in \operatorname{End}\left(A^{+} / S^{+}\right)
$$


where $k \in \mathbf{N},\left(i_{1}, \ldots, i_{k}\right) \in \mathbf{N}^{k}$ and

$\varphi_{\left(w, i_{1}, \ldots, i_{k}\right)}: A^{+} / S^{+} \ni r+S^{+} \mapsto\left[\left[\ldots\left[\left[w, a_{j_{i_{1}}}\right], a_{j_{i_{2}}}\right], \ldots, a_{j_{i_{k}}}\right], r\right]+S^{+} \in A^{+} / S^{+}$

is an endomorphism of $A^{+} / S^{+}$, determines a group homomorphism. Then the set

$$
\left\{\varphi_{\left(w, i_{1}, \ldots, i_{k}\right)} \mid w \in K_{g}, k \in \mathbf{N} \text { and }\left(i_{1}, \ldots, i_{k}\right) \in \mathbf{N}^{k}\right\}
$$

is finite, every kernel $\operatorname{Ker} \varphi_{\left(w, i_{1}, \ldots, i_{k}\right)}$ is of finite index in $K_{g}$ and therefore we deduce that

$$
I:=\bigcap_{\substack{w \in K_{g} \\\left(i_{1}, \ldots, i_{k}\right) \in \mathbf{N}^{k}}} \operatorname{Ker} \varphi_{\left(w, i_{1}, \ldots, i_{k}\right)}
$$

is of finite index in $K_{g}$ (and consequently in $A$ ). Moreover, $I \leq S$ and

$$
[I, \underbrace{A, \ldots, A}_{k \text { times }}] \leq S
$$

for any $k \in \mathbf{N}$. Hence

$$
I_{0}:=I+\sum_{k=1}^{\infty}[I, \underbrace{A, \ldots, A}_{k \text { times }}]
$$

is an ideal of finite index in $A$ such that $I_{0} \leq S$.

Corollary 4 If $A$ is a Lie FC-ring, then, for every $a \in A$, there exists an ideal $I_{a}$ of finite index in A such that $\left[a, I_{a}\right]=0$.

The next proposition is an analogue of [2, Theorem 5.2].

Proposition 3 Let $A$ be a Lie ring. Then the set $\operatorname{IDer} A$ is finite if and only if $\mid A$ : $Z(A) \mid<\infty$.

Proof $(\Rightarrow)$ Suppose that IDer $A=\left\{\operatorname{ad}_{u_{i}} \mid i=1, \ldots, m\right\}$ for some $m \in \mathbf{N}$ and $u_{1}, \ldots, u_{m} \in A$. If $x \in A$, then there exists $s=s(x) \in \mathbf{N}$ such that $1 \leq s \leq m$ and $\operatorname{ad}_{x}=\operatorname{ad}_{u_{s}}$. Hence $x \in u_{s}+Z(A)$ and $A / Z(A)=\left\{u_{i}+Z(A) \mid i=1, \ldots, m\right\}$ is finite.

$(\Leftarrow)$ Since

$$
A / Z(A)=\left\{a_{1}+Z(A), \ldots, a_{n}+Z(A)\right\}
$$

for some $n \in \mathbf{N}$ and $a_{1}, \ldots, a_{n} \in A$ and, for every $x \in A$, there exists $i=i(x)$ $(1 \leq i \leq n)$ such that $x \in a_{i}+Z(A)$, we see that $\operatorname{ad}_{x}=\operatorname{ad}_{a_{i}}$. Thus

$$
\operatorname{IDer} A=\left\{\operatorname{ad}_{x} \mid x \in A\right\}=\left\{\operatorname{ad}_{a_{i}} \mid i=1, \ldots, n\right\}
$$

is finite. 
Corollary 5 Let A be a Lie ring. Then the following hold:

(1) if $|A: Z(A)|<\infty$, then the commutator ideal $A^{\prime}$ is finite,

(2) if IDer $A$ is finite, then the commutator ideal $A^{\prime}$ is finite.

Proof For a proof, see [2, Lemma 5.12].

Lemma 6 Let A be a Lie ring. Then the following hold:

(1) if $A^{\prime}$ is finite, then $A$ is $F C$,

(2) if IDer $A$ is finite, then $A$ is $F C$.

Proof (1) Let $a \in A$. Since $\operatorname{ad}_{a}$ is an endomorphism of $A^{+}$and $Z(A) \leq C_{A}(x)=$ Ker ad ${ }_{a}$, we conclude that

$$
A / C_{A}(a)=A / \operatorname{Ker} \operatorname{ad}_{a} \cong[A, a] \leq A^{\prime}
$$

is finite for any $a \in A$. Hence $A$ is $F C$.

(2) follows immediately from part (1).

Lemma 7 If $A$ is a finitely generated Lie FC-ring, then its commutator ideal $A^{\prime}$ is finite.

Proof Suppose that $A$ is generated by some elements $x_{1}, \ldots, x_{n} \in A$. Inasmuch as $\left|A: C_{A}\left(x_{i}\right)\right|<\infty$ for $i=1, \ldots, n$ and

$$
Z(A)=\bigcap_{i=1}^{n} C_{A}\left(x_{i}\right),
$$

we have that $Z(A)$ is of finite index in $A$ and, by Corollary $5, A^{\prime}$ is finite.

Lemma 8 If $F$ is a finite ideal of a Lie ring $A$, then $\left|A: C_{A}(F)\right|<\infty$.

Proof Suppose that $F=\left\{x_{1}, \ldots, x_{n}\right\}$. Then

$$
\left[x_{i}, A\right] \cong A / C_{A}\left(x_{i}\right)
$$

for any $x_{i} \in F$ what implies that

$$
\bigcap_{i=1}^{n} C_{A}\left(x_{i}\right) \leq C_{A}(F)
$$

and the result follows.

Recall that $M$ is a minimal ideal of a Lie ring $A$ if $M \neq 0$ and, for any ideal $I$ of $A$, the implication

$$
0 \leq I \leq M \Rightarrow I=0 \text { or } I=M
$$

holds. If $M$ is a minimal nonzero ideal of $A$, then $[M, M]=M$ (i.e. $M$ is perfect) or $[M, M]=0$. 
Lemma 9 If $M$ is a perfect minimal ideal of a Lie ring $A$, then the quotient Lie ring $A / C_{A}(M)$ is prime.

Proof If $B, C$ are ideals of $A$ such that $[B, M] \neq 0$ and $[C, M] \neq 0$, then $[B, M]=$ $M=[C, M]$ and $M \leq[B, C]$. This yields that $[[B, C], M] \neq 0$ and so $A / C_{A}(M)$ is prime.

Lemma 10 If $F$ is a finite ideal of a Lie ring A, then the following hold:

(1) if $F$ is a perfect minimal ideal, then $A=F \oplus C_{A}(F)$ is a direct sum of ideals,

(2) if $F$ does not contain nonzero nonabelian ideal of $A$, then there exist perfect minimal ideals $B_{1}, \ldots, B_{k}$ of $A$ such that $B_{i} \leq F(i=1, \ldots k), A=B_{1} \oplus \cdots \oplus$ $B_{k} \oplus C$ is a direct sum of ideals and $F \cap C$ is solvable,

(3) $F$ contains a solvable ideal $S$ of $A$ such that $F=F^{\prime}+S$ and the quotient Lie $\operatorname{ring} A / S=(F / S) \oplus K$ is a direct sum for some its ideal $K$.

Proof (1) By Lemmas 9 and $8, K:=A / C_{A}(F)$ is a finite prime (and therefore simple) Lie ring. Since $K$ is perfect and $F \nsubseteq C_{A}(F)$, we deduce that

$$
\left(F+C_{A}(F)\right) / C_{A}(F)=A / C_{A}(F)
$$

and the result follows.

(2) It is easy to see that $F$ contains a minimal ideal $B_{1}$ of $A$ and $B_{1}$ is nonabelian. By part (1), $A=B_{1} \oplus C_{A}\left(B_{1}\right)$ is a direct sum of ideals. Since $F$ is finite, we obtain the assertion by finite number of steps.

(3) Suppose that $S$ is an ideal generated by all solvable ideals of $A$ that are contained in $F$. Then $F / S$ is a finite semiprime Lie ring (and consequently it is a direct sum of finitely many nonabelian minimal ideals of $A / S$ ) in view of part (1). This gives that $F=F^{\prime}+S$. The rest it follows from part (2).

Corollary 6 Let $A$ be a Lie ring. If IDer $A$ is finite, then the commutator ideal $A^{\prime}$ is finite and there exists a solvable ideal $S$ of $A$ such that $S \leq A^{\prime}, A^{\prime}=A^{\prime \prime}+S$ and $A / S=\left(A^{\prime} / S\right) \oplus K$ is a direct sum of ideals for some abelian ideal $K$.

Proof By Proposition 3 and Corollary 5, $A^{\prime}$ is finite and so the result holds by Lemma 10.

\section{Generalized derivations}

Let

$$
\operatorname{CDer} A:=\{h \in \operatorname{Der} A \mid h(A) \subseteq Z(A)\}
$$

be the set of all central derivations of $A$. The structural properties of a Lie algebra $L$ with central inner derivations (i.e. $\operatorname{ad} L \subseteq \operatorname{CDer} L$ ) was studied by Tôgô [30].

Lemma 11 Let A be a Lie ring. Then: 
(i) GDer $A$ is a Lie ring,

(ii) $F(Z(A)) \subseteq Z(A)$ for any $F \in \operatorname{GDer} A$,

(iii) $\operatorname{CDer} A$ is an ideal of $\operatorname{GDer} A$,

(iv) $\operatorname{GDer} A=\mathrm{M}(A)+\operatorname{Der} A$, where $\mathrm{M}(A)$ is an ideal of $\mathrm{GDer} A$, and

$$
\mathrm{M}(A) \bigcap \operatorname{Der} A \subseteq \operatorname{CDer} A
$$

(v) $\operatorname{IGDer} A=\mathrm{M}(A)+\operatorname{ad} A$, where $\mathrm{M}(A)$ is an ideal of IGDer $A$, and

$$
\mathrm{M}(A) \bigcap \operatorname{ad} A \subseteq \operatorname{CDer} A,
$$

(vi) if $B$ is a $D$-closed ideal of $A$, then

$\mathrm{I}_{\mathrm{B}} \mathrm{GDer} A:=\left\{F \in \mathrm{GDer} A \mid F\right.$ is associated with some $\operatorname{ad}_{a}$, where $\left.a \in B\right\}$

(in particular, $\mathrm{M}(A)=\mathrm{I}_{\mathrm{O}} \operatorname{GDer} A=\mathrm{I}_{\mathrm{Z}(\mathrm{A})} \mathrm{GDer} A \subseteq \mathrm{IGDer} A:=\mathrm{I}_{\mathrm{A}}$ GDer $A$ ) is an ideal of GDer $A$,

(vii) $C(A):=\{k \in \mathrm{M}(A) \mid k(A) \subseteq Z(A)\}$ is an ideal of GDer $A$,

(viii) if $(F, \delta),(F, \mu) \in \operatorname{GDer} A$, then $\delta+\operatorname{CDer} A=\mu+\operatorname{CDer} A$,

(ix) if $\left(F, \operatorname{ad}_{a}\right),\left(F, \operatorname{ad}_{b}\right) \in \operatorname{GDer} A$ for some $a, b \in A$, then $[a-b, A] \subseteq Z(A)$.

Proof Assume that $(F, \delta),(H, d) \in G, T \in \mathrm{M}(A), h \in \mathrm{CDer} A$ and $x, y \in A$.

(i) We see that $(F-H, \delta-d) \in G$,

$$
\begin{gathered}
{[F, H]([x, y])=F([H(x), y]+[x, d(y)])-H([F(x), y]+[x, \delta(y)])} \\
=[[F, H](x), y]+[x,[\delta, d](y)]
\end{gathered}
$$

and so $([F, H],[\delta, d]) \in G$.

(ii) Evident.

(iii) Since $h(A) \subseteq Z(A)$ for $h \in \operatorname{CDer} A$, we have that $[F, h](A) \subseteq Z(A)$, i.e. $[F, h] \in \operatorname{CDer} A$.

(iv) The equality

$$
[F, T]([x, y])=[[F, T](x), y]
$$

implies that $[F, T] \in \mathrm{M}(A)$ and so $\mathrm{M}(A)$ is an ideal of $G$. Moreover,

$$
(\delta-F)([x, y])=[\delta(x), y]+[x, \delta(y)]-[F(x), y]-[x, \delta(y)]=[(\delta-F)(x), y]
$$

and thus $\delta-F \in \mathrm{M}(A)$. If $h \in D \cap \mathrm{M}(A)$, then

$$
[h(x), y]=h([x, y])=[h(x), y]+[x, h(y)] .
$$

From this it follows $[x, h(y)]=0$ and therefore $h(A) \subseteq Z(A)$.

(v) By the same argument as in (iv). 
(vi) If $\left(K, \operatorname{ad}_{a}\right),\left(M, \operatorname{ad}_{b}\right) \in \mathrm{I}_{\mathrm{B}} \operatorname{GDer} A$, then $\left(K-M, \operatorname{ad}_{a-b}\right) \in \mathrm{I}_{\mathrm{B}} \operatorname{GDer} A$ and

$$
\begin{aligned}
{[F, K]([x, y]) } & =F\left([K(x), y]+\left[x, \operatorname{ad}_{a}(y)\right]\right)-K([F(x), y]+[x, \delta(y)])= \\
& =[[F, K](x), y]+\left[x, \operatorname{ad}_{\delta(a)}(y)\right]
\end{aligned}
$$

that is $\left([F, K], \operatorname{ad}_{\delta(a)}\right) \in \mathrm{I}_{\mathrm{B}} \operatorname{GDer} A$.

(vii) If $k \in C(A)$, then

$$
[F, k]([x, y])=[[F, k](x), y]=0
$$

and consequently $[F, k] \in C(A)$.

(viii)-(ix) If $(F, \delta),(F, \mu) \in G$ for some $\delta, \mu \in D$, then

$$
[x, \delta(y)]=[x, \mu(y)]
$$

and therefore $[x,(\delta-\mu)(y)]=0$. This means that $(\delta-\mu)(A) \subseteq Z(A)$ and the result follows.

Corollary 7 Let A be a Lie ring. Then the following hold:

(1) if $Z(A)=0$, then

$$
\operatorname{GDer} A=\mathrm{M}(A)+\operatorname{Der} A, \operatorname{IGDer} A=\mathrm{M}(A)+\operatorname{ad} A \text { and } \mathrm{M}(A) \bigcap \operatorname{Der} A=0,
$$

(2) if $A$ is a simple (respectively semiprime or prime) ring, then the Lie rings $\operatorname{GDer} A / \mathrm{M}(A)$ and $\operatorname{Der} A$ are isomorphic.

Proof (1) If $Z(A)=0$, then $\operatorname{CDer} A=0$ and the result holds by Lemma 11 (iv) and (v).

(2) Since $Z(A)$ is an ideal of $A$, we deduce that $Z(A)=0$. The rest follows in view of part (1).

Let $\Phi \subseteq \operatorname{GDer} A, \Gamma \subseteq \operatorname{Der} A$,

$$
\begin{aligned}
T_{\Phi} & =\{d \in \operatorname{Der} A \mid \text { there is } H \in \Phi \text { that is associated with } d \in \operatorname{Der} A\} \\
U_{\Gamma} & =\{H \in \operatorname{GDer} A \mid H \text { is associated with some } d \in \Gamma\}
\end{aligned}
$$

and

$$
\Sigma_{\Phi}=\left\{a \in A \mid \text { there exists } H \in \Phi \text { that is associated with } \operatorname{ad}_{a}\right\}
$$

Lemma 12 Let A be a Lie ring. Then the following hold:

(i) if $\Phi$ is an ideal of GDer $A$, then $T_{\Phi}$ is an ideal of $\operatorname{Der} A$, 
(ii) if $\Gamma$ is an ideal of $\operatorname{Der} A, U_{\Gamma}$ is a nonzero ideal of GDer $A$ (in particular, $U_{0}=$ $\mathrm{M}(A))$,

(iii) if $\Phi$ is an ideal of IGDer $A$ (respectively GDer $A$ ), then $\Sigma_{\Phi}$ is an ideal (respectively a D-ideal) of $A$.

Proof For a proof, see [2, Lemma 5.7].

Lie algebras $L$ with abelian derivation algebras $\operatorname{Der} L$ was studied, in particular, in [29].

Lemma 13 Let $A$ be a Lie ring and $(F, d) \in \operatorname{GDer} A$. Then we have:

(i) if $F=0$, then $d(A) \subseteq Z(A)$,

(ii) if $d(A) \subseteq Z(A)$, then $F \in \mathrm{M}(A)$,

(iii) if GDer $A$ is an abelian Lie ring, then $\operatorname{Der} A$ is abelian,

(iv) if $A \neq 0$, then $\mathrm{M}(A) \neq 0$.

Proof For a proof, see [2, Lemma 5.4].

Lemma 14 Let $A$ be a Lie ring and $\left(M, \operatorname{ad}_{a}\right) \in \operatorname{IGDer} A$. Then the following hold:

(i) if $M=0$, then $[a, A] \subseteq Z(A)$,

(ii) if $[a, A] \subseteq Z(A)$, then $M \in \mathrm{M}(A)$,

(iii) if IGDer $A$ is an abelian Lie ring, then $\operatorname{ad} A$ is abelian,

(iv) if $A$ is abelian, then $\operatorname{IGDer} A=\mathrm{M}(A)$.

Proof For a proof, see [2, Lemmas 5.4 and 5.5].

Lemma 15 Let $A$ be a Lie ring, $B$ its ideal. Then:

(i) if $\Phi$ is an ideal of $\operatorname{GDer} A$, then $\Phi \cap \operatorname{IGDer} A=0$ implies that $[\delta(A), A] \subseteq Z(A)$ for any $\delta \in T_{\Phi}$,

(ii) the following conditions are equivalent:

(a) $\mathrm{I}_{\mathrm{B}} \mathrm{GDer} A \subseteq \mathrm{M}(A)$,

(b) $B \subseteq Z(A)$,

(c) $\operatorname{ad}_{B} A=0$,

(iii) there exist Lie ring isomorphisms:

(d)

$\operatorname{Der} A / \operatorname{CDer} A \ni \delta+\operatorname{CDer} A \mapsto \delta+\mathrm{M}(A) \in \operatorname{GDer} A / \mathrm{M}(A)$,

(e)

$$
\begin{aligned}
& \operatorname{ad} A /(\operatorname{ad} A \bigcap \operatorname{CDer} A) \ni \operatorname{ad}_{a}+(\operatorname{ad} A \bigcap \operatorname{CDer} A) \mapsto \operatorname{ad}_{a} \\
& \quad+\mathrm{M}(A) \in \operatorname{IGDer} A / \mathrm{M}(A)
\end{aligned}
$$

Proof (i) If $(F, \delta) \in \Phi$ and $\left(H, \operatorname{ad}_{a}\right) \in \operatorname{IGDer} A$, then $\left([F, H], \operatorname{ad}_{\delta(a)}\right) \in \operatorname{IGDer} A$ and so $\operatorname{ad}_{\delta(a)} \in \operatorname{CDer} A$ by Lemma 14 (i).

(ii)-(iii) are evident. 
Corollary 8 Let $A$ be a Lie ring. If ad $A$ is a semiprime (respectively prime or simple) Lie ring, then $\operatorname{IGDer} A / \mathrm{M}(A)$ is a semiprime (respectively prime, or simple) Lie ring.

Proof If $a \in A$ and $\operatorname{ad}_{b} \in \operatorname{CDer} A \cap \operatorname{ad} A$, then $[a, b] \in Z(A)$ and $\left[\operatorname{ad}_{a}, \operatorname{ad}_{b}\right]=$ $\operatorname{ad}_{[a, b]}=0$. Then $\operatorname{CDer} A \cap \operatorname{ad} A=0$ because $\operatorname{ad} A$ is semiprime (respectively prime or simple) and so $\operatorname{ad} A$ is isomorphic to $\operatorname{IGDer} A / \mathrm{M}(A)$ by Lemma 15 (iii).

Lemma 16 Let $A$ be a nonnilpotent Lie ring. If $A$ is primary, then the quotient Lie ring so is $\operatorname{IGDer} A / \mathrm{M}(A)$.

Proof Assume that $\Phi, \Lambda$ are ideals of IGDer $A$ such that $[\Phi, \Lambda]=0$. By Lemma 14 (i), [[ $\left.\left.\Sigma_{\Phi}, \Sigma_{\Lambda}\right], A\right] \subseteq Z(A)$. Since $A$ is nonnilpotent primary (and therefore $Z(A)=0$ ), we deduce that $\left[\Sigma_{\Phi}, \Sigma_{\Lambda}\right]=0$. This implies that $\Sigma_{\Phi}=0$ (and then $\Phi \subseteq \mathrm{M}(A)$ ) or

$$
[\underbrace{\Sigma_{\Lambda}, \ldots, \Sigma_{\Lambda}}_{m \text { times }}]=0
$$

(and consequently

$$
[\underbrace{\Lambda, \ldots, \Lambda}_{m \text { times }}] \subseteq \mathrm{M}(A)
$$

for some positive integer $m$. Hence $\operatorname{IGDer} A / \mathrm{M}(A)$ is a primary Lie ring.

\section{Proofs}

Proof of Proposition 1. (1) Let $D$ be a simple Lie ring. Then $D$ and $A$ are nonzero. Since $\operatorname{ad} A$ is an ideal of $D$, we deduce that $\operatorname{ad} A=0$ (and then $A$ is abelian) or $\operatorname{ad} A=D$. Assume that $\operatorname{ad} A=D$ and $K$ is arbitrary noncentral ideal of $A$. Then

$$
0 \neq \operatorname{ad}_{K} A=\operatorname{ad} A
$$

by Lemma 1 (ii) and so $A=K+Z(A)$. This means that $\bar{A}:=A / Z(A) \cong \operatorname{ad} A$ is a simple Lie ring. Then it is nonabelian and therefore $\bar{A}^{\prime} \neq \overline{0}$. Consequently $\bar{A}^{\prime}=\bar{A}$ and $A=A^{\prime}$ is simple or Eq. (2) follows.

(2) Let $A$ be a $D$-simple Lie ring. Then $0 \neq A^{\prime}=A$ and $Z(A)=0$. By the same argument, as in the proof of Theorem 1.1 (i) from [27], $D=\operatorname{ad} A$ is complete.

Proof of Theorem 1. (a) Let $A$ be a $D$-prime Lie ring. Then $Z(A)=0$. Assume that $\Phi, \Lambda$ are nonzero ideals of $D$ such that $[\Phi, \Lambda]=0$. By Lemma 4 ,

$$
\Phi_{1}:=\Phi \bigcap \operatorname{ad} A \neq 0 \text { and } \Lambda_{1}:=\Lambda \bigcap \operatorname{ad} A \neq 0
$$

and $\nabla_{\Phi_{1}}, \nabla_{\Lambda_{1}}$ are nonzero. Since

$$
\operatorname{ad}_{\left[\nabla_{\Phi_{1}}, \nabla_{\Lambda_{1}}\right]} A=\left[\operatorname{ad}_{\nabla_{\Phi_{1}}} A, \operatorname{ad}_{\nabla_{\Lambda_{1}}} A\right]=\left[\Phi_{1}, \Lambda_{1}\right]=0
$$


we see that

$$
\left[\nabla_{\Phi_{1}}, \nabla_{\Lambda_{1}}\right] \subseteq Z(A)=0
$$

By Lemma 1 (iii), $\nabla_{\Phi_{1}}$ and $\nabla_{\Lambda_{1}}$ are $D$-ideals of $A$ and we obtain a contradiction. Hence $D$ is prime.

(b) If $A$ is a $D$-semiprime Lie ring, then we can obtain that $D$ is semiprime by the same argument as in part (a).

Proof of Theorem 2. (a) Assume that $G / \mathrm{M}(A)$ is a prime Lie ring and $B, C$ are $G$ ideals of $A$ such that

$$
[B, C] \subseteq Z(A)
$$

Then $\mathrm{I}_{\mathrm{B}}$ GDer $A, \mathrm{I}_{\mathrm{C}}$ GDer $A$ are ideals of $G$ by Lemma 11 (vi) and

$$
\left[\mathrm{I}_{\mathrm{B}} \operatorname{GDer} A, \mathrm{I}_{\mathrm{C}} \mathrm{GDer} A\right] \subseteq \mathrm{M}(A)
$$

in view of Eq. (6). Then, by the primeness of $G / \mathrm{M}(A), \mathrm{I}_{\mathrm{B}} \mathrm{GDer} A \subseteq \mathrm{M}(A)$ or $\mathrm{I}_{\mathrm{C}} \mathrm{GDer} A \subseteq \mathrm{M}(A)$ what implies that $B \subseteq Z(A)$ or $C \subseteq Z(A)$ by Lemma 15 (iii), and hence $Z(A)$ is $G$-prime.

(b) If $G / \mathrm{M}(A)$ is a semiprime Lie ring, then we can prove by the same argument as in case (a).

(c) Assume that $G / \mathrm{M}(A)$ is a simple Lie ring and $B$ is a $G$-ideal of $A$. Then $\mathrm{I}_{\mathrm{B}} \mathrm{GDer} A$ is an ideal of $G$ and consequently

$$
\mathrm{I}_{\mathrm{B}} \mathrm{GDer} A \subseteq \mathrm{M}(A)
$$

(and so $B \subseteq Z(A)$ by Lemma 15 (ii)) or

$$
G / \mathrm{M}(A)=\mathrm{I}_{\mathrm{B}} \mathrm{GDer} A / \mathrm{M}(A) .
$$

In the second case we have $\mathrm{M}(A) \neq \mathrm{I}_{\mathrm{B}} \operatorname{GDer} A=\operatorname{IGDer} A=\operatorname{GDer} A$. Then $\operatorname{ad}_{B} A=$ $\operatorname{ad} A$ what gives that $A=B+Z(A)$. This means that $Z(A)$ is $G$-simple.

(d) Let $G / \mathrm{M}(A)$ be a primary Lie ring and $B, C$ be $G$-ideals of $A$ such that Eq. (7) is true. Then Eq. (8) is true (and so $\mathrm{I}_{\mathrm{B}} \mathrm{GDer} A \subseteq \mathrm{M}(A)$ ) or

$$
[\underbrace{\left[\mathrm{I}_{\mathrm{C}} \mathrm{GDer} A, \ldots, \mathrm{I}_{\mathrm{C}} \mathrm{GDer} A\right.}_{m \text { times }}] \subseteq \mathrm{M}(A)
$$

for some positive integer $m$ ). Then $B \subseteq Z(A)$ or

$$
[\underbrace{C, \ldots, C}_{m \text { times }}] \subseteq Z(A)
$$

and consequently $Z(A)$ is a $G$-primary ideal of $A$.

Acknowledgements The author wishes to thank the referee for useful remarks and suggestions. 
Open Access This article is distributed under the terms of the Creative Commons Attribution 4.0 International License (http://creativecommons.org/licenses/by/4.0/), which permits unrestricted use, distribution, and reproduction in any medium, provided you give appropriate credit to the original author(s) and the source, provide a link to the Creative Commons license, and indicate if changes were made.

\section{References}

1. Angelopoulus, E.: Algébres de Lie satisfaisant $\mathfrak{g}=[\mathfrak{g}, \mathfrak{g}], \operatorname{Der}(\mathfrak{g})=\operatorname{adg}$. C.R. Acad. Sci. Paris Sér. I Math. 306, 523-525 (1988)

2. Artemovych, O.D., Blackmore, D., Prykarpatsky, A.K.: Poisson brackets, Novikov-Leibniz structures and integrable Riemann hydrodynamic systems. J. Nonlinear Math. Phys. 24, 41-72 (2017)

3. Block, R.: Determinations of the differentiably simple rings with a minimal ideal. Ann. Math. 90, 433-459 (1969)

4. Brešar, M.: On the distance of the composition of two derivations to the generalized derivations. Glasgow J. Math. 33, 89-93 (1991)

5. de Ruiter, D.: On derivations of Lie algebras. Compositio Math. 28, 299-303 (1974)

6. Dixmier, J., Lister, W.G.: Derivations of nilpotent Lie algebras. Proc. Am. Math. Soc. 8, 155-158 (1957)

7. Elduque, A., Montaner, F.: A note on derivations of simple algebras. J. Algebra 165, 636-644 (1994)

8. Fialkow, L.A.: Generalized derivations, In: Topics in Modern Operator Theory (Timişoara/Herculane, 1980). Operator Theory: Advances and Applications, vol. 2, pp. 95-103. Birkhäuser, Basel-Boston (1981)

9. Hochschild, G.: Semi-simple algebras and generalized derivations. Am. J. Math. 64, 677-694 (1942)

10. Hopkins, N.C.: Generalized derivations of nonassociative algebras. Nova J. Math. Game Theory Algebra 5, 215-224 (1996)

11. Jacobson, N.: Derivations algebras and multiplication algebras of semi-simple Jordan algebras. Ann. Math. 50, 866-874 (1949)

12. Jacobson, N.: A note on automorphisms and derivations of Lie algebras. Proc. Am. Math. Soc. 6, 281-283 (1955)

13. Jacobson, N.: Lie Algebras. Wiley, New York (1962)

14. Jacobson, N.: Structure and Representations of Jordan Algebras, vol. 39. American Mathematical Society Colloquium Publications, Providence (1968)

15. Kac, V.G.: Infinite Dimensional Lie Algebras, 3rd edn. Cambridge University Press, Cambridge (1994)

16. Leger, G.: Derivations of Lie algebras. III. Duke Math. J. 30, 637-645 (1963)

17. Leger, G.F., Luks, E.M.: Generalized derivations of Lie algebras. J. Algebra 228, 165-203 (2000)

18. Lewin, J.: Subrings of finite index in finitely generated rings. J. Algebra 5, 84-88 (1967)

19. Liao, J., Zheng, D., Liu, H.: Towers of derivation for Lie rings and some results on complete Lie rings. Acta Math. Sci. 30B, 1769-1775 (2010)

20. Luks, E.M.: Lie algebras with only inner derivations need not be complete. J. Algebra 15, 280-282 (1970)

21. Meng, D.J., Wang, S.P.: On the construction of complete Lie algebras. J. Algebra 176, 621-637 (1995)

22. Robinson, D.J.S.: A Course in the Theory of Groups. Graduate texts in mathematics, vol. 80. Springer, New York Berlin (1982)

23. Sato, T.: The derivations of the Lie algebras. Tohoku Math. J. 23, 21-36 (1971)

24. Schafer, R.D.: Inner derivations of non-associative algebras. Bull. Am. Math. Soc. 55, 769-776 (1949)

25. Schenkman, E.: On the derivation algebra and holomorph of a nilpotent Lie algebras. Mem. Am. Math. Soc. 14, 15-22 (1955)

26. Stitzinger, E.L.: On Lie algebras with only inner derivations. J. Algebra 105, 341-343 (1987)

27. Su, Y., Zhu, L.: Derivations algebras of centerless perfect Lie algebras. J. Algebra 285, 508-515 (2005)

28. Tôgô, S.: On the derivations of Lie algebras. J. Sci. Hiroshima Univ. Ser. A 19, 71-77 (1955)

29. Tôgô, S.: On the derivation algebras of Lie algebras. Canad. J. Math. 13, 201-216 (1961)

30. Tôgô, S.: Derivations of Lie algebras. J. Sci. Hiroshima Univ. Ser. A-I 28, 133-158 (1964)

31. Tôgô, S.: Lie algebras which have few derivations. J. Sci. Hiroshima Univ. Ser. A-I 29, 29-41 (1965)

32. Vinberg, È.B.: Generalized derivations of Lie algebras. In: Algebra and Analysis (Irkutsk, 1989), Amer. Math. Soc. Trans. Ser. 2, vol. 163, pp. 185-188. American Mathematical Society, Providence (1995) 
33. Zhevlakov, K.A., Slinko, A.M., Shestakov, I.P., Shirshov, A.I.: Rings that are Nearly Associative, translated from Russian by Harry F. Smoth. Academic Press, New York (1982)

34. Walcher, S.: On derivations of simple algebras. Algebras Groups Geom. 4, 379-382 (1987)

35. Zassenhaus, H.: Über Lie'sche Ringe mit Primzahlcharakteristik. Abh. Math. Sem. Univ. Hamburg 13, $1-100$ (1939)

Publisher's Note Springer Nature remains neutral with regard to jurisdictional claims in published maps and institutional affiliations. 